\title{
The future of the Quality and Outcomes Framework in England
}

It's time to rethink one of the most ambitious experiments in general practice

Martin Marshall, Professor of Healthcare Improvement, UCL

Martin Roland, Emeritus Professor of Health Services Research, University of Cambridge

To describe the Quality and Outcomes Framework (QOF) - one of the most ambitious payfor-performance schemes introduced into any health system - as divisive would be an understatement. Launched in the UK in 2004 as a way of encouraging adherence to specified evidence-based elements of general practice care, QOF has changed the nature of general practice [1] in ways that have polarised those who work in the sector and external commentators.

Advocates highlight how patient care, especially for those with long term conditions, is now more structured, more systematic and more likely to be based on high quality research evidence. They point out the rapid progress with computerisation that followed the implementation of the initiative, how better data are being used in more effective ways, and how work has been distributed more efficiently between a larger and more diverse primary care workforce. And they celebrate the extent to which these improvements have taken place across nearly every practice in the country.

Others have expressed concerns about both the intended and unintended consequences of QOF. Critics claim that using financial incentives to focus attention on technical aspects of care that are easily measured has diverted attention from inter-personal elements that are less easily objectified. They say that clinical care for single diseases has been prioritised over holistic care for patients with multiple health problems. They describe how doctors have been de-professionalised by a box-ticking and computer prompt-driven culture and how biomedical and policy agendas have been given a higher priority than what is important to patients. And they claim that the potential of QOF to incentivise continuous improvement is limited given that many practices achieved maximum remuneration within a year of the scheme's introduction.

Whilst many changes have been described to structures and working processes, a big question is whether QOF has improved outcomes for patients. The evidence is far from convincing. Observational studies suggest modest improvements in some aspects of clinical care $[2,3]$, a small reduction in the rate of increase of emergency admissions for incentivised 
conditions [4] but no clear effect on overall mortality.[5] There is little empirical evidence that QOF impacts negatively on the coordination or integration of care, the provision of holistic care, or patient experience but little sign of benefit either.[6] These findings are consistent with evidence from other parts of the world that financial incentives are often less effective than those who bring them in expect.[7]

QOF has become increasingly unpopular with GPs, partly because of the administrative demands of the scheme at a time of rising workload, and partly because of indicators introduced in the last few years that were poorly evidence based or seemed to address a managerial rather than a clinical agenda. Inevitably, questions are being asked about its future. In 2015 the Royal College of General Practitioners called for it to be replaced by a system of payment that encouraged a more holistic approach to patient care.[8] In 2016 QOF was abolished in Scotland and replaced by a requirement for GPs to take part in local peer led quality improvement activities (GP Quality Clusters).[9] Some localities in England have replaced QOF with local incentive schemes and earlier this year NHS England said that it was committed in principle to removing QOF altogether.[10] The British Medical Association, however, have called for it to be retained, in part because of the likely disruption and the risk to practice income if it were abolished.

We believe that QOF should only be changed if whatever replaces it is better for patients, for individual practices and for the future sustainability of general practice as a specialty. There are a number of principles that should guide any replacement. First, there may be a case for retaining a limited number of indicators with clear relationships to health outcome - or at least measuring these to ensure that quality does not decline when incentives are removed. Second, whatever replaces QOF must build on the professionalism and good will that still exists in general practice: Scotland has made a bold statement of confidence in the profession and England could be equally courageous. Third, in line with the recently published position statement on quality from the Royal College of General Practitioners [11], new arrangements should place a greater emphasis on encouraging a process of continuous improvement in care rather than the attainment of standards. A focus on these last two will recognise the value of the more complex activities that general practice must do, like managing uncertainty, engaging with the social determinants of health and encouraging shared decision making with patients.

Fifteen years ago we asked whether QOF would be the renaissance or the requiem for general practice.[12] It has been neither. It has resulted in some benefits and has caused 
some harm. Policy makers and the profession now have an opportunity move on and do better.

803 words

Declarations of interest: Professor Martin Marshall is Vice Chair of the Royal College of General Practitioners. Professor Roland advised the Department of Health and British Medical Association during the development of the Quality and Outcomes Framework from 2001 to 2003.

1. Roland M, Guthrie B. The Quality and Outcomes Framework. How did QOF come about and what have we learned? BMJ 2016; 354: i4060 www.bmj.com/content/bmj/354/bmj.i4060.full.pdf

2. Campbell SM, Reeves D, Kontopantelis E, Sibbald B, Roland M. Effects of pay for performance on the quality of primary care in England. $\mathrm{N}$ Engl $\mathrm{J}$ Med 2009;361:368-78.

3. Langdown $\mathrm{C}$, and Peckham S. The use of financial incentives to help improve health outcomes: is the quality and outcomes framework fit for purpose? A systematic review. J Public Health (Oxf). 2014 Jun;36(2):251-8

4. Harrison MJ, Dusheiko M, Sutton M, Gravelle H, Doran T, Roland M. Effect of a national primary care pay for performance scheme on emergency hospital admissions for ambulatory care sensitive conditions: controlled longitudinal study. BMJ 2014; 349: g6423

5. Ryan AM, Krinsky S, Kontopantelis E, Doran T. Long-term evidence for the effect of pay-for-performance in primary care on mortality in the UK: a population study. Lancet. 2016 Jul 16;388(10041):268-74.

6. Forbes L, Marchand C, Doran T, Peckham S. The role of the Quality and Outcomes Framework in the care of long-term conditions: a systematic review $\mathrm{Br} \mathrm{J}$ Gen Pract 25 September 2017; bjgp17X693077.

7. Eijkenaar F, Emmert M, Scheppach M, Schöffski O. Effects of pay for performance in health care: a systematic review of systematic reviews. Health Policy 2013;110:115-30.

8. Royal College of General Practitioners. Blueprint for Building the new deal for general practice. RCGP London 2015. http://www.rcgp.org.uk/policy/rcgp-policyareas/blueprint-for-general-practice.aspx (Accessed 8th October 2017) 
9. Health Improvement Scotland and NHS Scotland. Driving and Supporting Improvement in Primary Care 2016-2020. Health Improvement Scotland 2016. www.healthcareimprovementscotland.org/our_work/primary_care/programme_resour ces/primary_care_approach.aspx (Accessed 8th October 2017)

10. NHS England. General practice forward view, 2016. https://www.england.nhs.uk/gp/gpfv (Accessed 8th October 2017)

11. Royal College of General Practitioners. RCGP position statement on quality in general practice. RCGP. London. 2017 RCGP-Position-statement-Quality-ingeneral-practice-2017.pdf (Accessed $8^{\text {th }}$ October 2017)

12. Marshall $M$, Roland $M$. The new contract: renaissance or requiem for general practice? British Journal of General Practice 2002; 52: 531-2 